\title{
Mechanismen der Signalbildung an gassensitiven Elektroden: ITO vs. Pt
}

\author{
Stefan Dietrich ${ }^{1}$, Mihails Kusnezoff ${ }^{1}$ und Sindy Mosch ${ }^{1}$ \\ ${ }^{1}$ Fraunhofer-Institut für Keramische Technologien und Systeme IKTS, Dresden, Deutschland \\ Kontakt: stefan.dietrich@ikts.fraunhofer.de
}

\section{Einleitung}

Potentiometrische Gassensoren auf Basis Sauerstoffionen-leitender Festelektrolyte haben sich in den vergangenen Jahrzehnten vor allem in der Überwachung von Kraftfahrzeug-Abgasen und der Steuerung industrieller Verbrennungsprozesse bewährt. Die Sensoren basieren in der Regel auf Zirkoniumdioxid $\left(\mathrm{ZrO}_{2}\right)$, dessen lonenleitfähigkeit durch Dotierungen mit Metalloxiden wie beispielsweise Yttriumoxid $\left(\mathrm{Y}_{2} \mathrm{O}_{3}\right)$ sowie Scandium $\left(\mathrm{Sc}_{2} \mathrm{O}_{3}\right)$ erhöht wird. Als Elektrodenwerkstoff wird aufgrund der hohen katalytischen Aktivität und der chemischen und Hochtemperatur-Beständigkeit üblicherweise Platin eingesetzt. Neben der Bestimmung der Sauerstoffkonzentration in Gasgemischen eignet sich dieser Sensortyp unter Ausnutzung der sogenannten Mischpotentialbildung jedoch auch zur Detektion brennbarer oder toxischer Gase wie Wasserstoff, Stickstoffdioxid oder Kohlenmonoxid. Empfindlichkeit und Selektivität des Sensors hängen dabei jedoch stark von der Wahl der verwendeten Elektrodenwerkstoffe ab. Der Einfluss des Elektrodenwerkstoffs auf die Signalbildung ist in den vergangenen Jahrzehnten dank erheblicher Anstrengungen der internationalen Forschung und Entwicklung zwar für die standardmäßig eingesetzten PlatinMesselektroden umfangreich untersucht worden, für andere aussichtsreiche Werkstoffklassen wie beispielsweise Metalloxide jedoch noch nicht ausreichend verstanden. Ohne die Kenntnis der fundamentalen Wechselwirkungsmechanismen ist die gezielte Entwicklung und Optimierung gassensitiver Elektroden auf Basis von Metalloxiden jedoch erheblich erschwert.

Zur Aufklärung dieser Wechselwirkungsmechanismen wurde daher ein Verfahren entwickelt, bei welchem neben Adsorptions-/Desorptionsprozessen an Pulverproben auch elektrische und elektrochemische Effekte an Funktionsschichten untersucht werden. Mit dem Verfahren wurden die signalbildenden Prozesse an Indium-Zinnoxid (ITO) und Platin (Pt) unter Einfluss von Kohlenmonoxid und weiterer Gasspezies charakterisiert und ein korrespondierendes Modell abgeleitet. Das verwendete IndiumZinnoxid (ITO) ist ein n-halbleitendes Mischoxid, welches aus Indium(III)Oxid $\left(\mathrm{In}_{2} \mathrm{O}_{3}\right)$ und Zinn(IV) Oxid $\left(\mathrm{SnO}_{2}\right)$ mit einem Massenverhältnis von typischerweise 90:10 besteht. Dünne ITO-Schichten weisen eine hohe Transmission im sichtbaren Teil des Lichtspektrums von bis zu 90\% auf. Darüberhinaus besitzt ITO eine vergleichsweise hohe elektrische Leitfähigkeit von bis zu $10^{4} \mathrm{~S} / \mathrm{cm}$, weshalb der Werkstoff einer der wichtigsten Vertreter der sogenannten transparenten leitfähigen Oxide (transparent conducting oxides, TCO) zählt. Als solches wird ITO bereits umfangreich für die Herstellung transparenter Elektroden für LCD-Bildschirme, in Touch Screens und als antistatische Beschichtung eingesetzt. In den vergangenen Jahrzehnten wurde ITO jedoch auch zunehmend als gassensitives Material für die Detektion von $\mathrm{CO}, \mathrm{CO}_{2}, \mathrm{H}_{2}, \mathrm{CH}_{4}, \mathrm{NH}_{3}, \mathrm{NO}$, $\mathrm{NO}_{2}$ und weiteren Gasen untersucht. Die meisten dieser Untersuchungen standen jedoch im Zusammenhang mit dem Einsatz in chemoresistiven Dünnschicht-Halbleitergassensoren, wohingegen der Einsatz als poröse Dickschicht bisher nur wenig dokumentiert ist [1-4].

\section{Methoden und Materialien}

Die Werkstoffcharakterisierung wurde bei allen Methoden im Temperaturbereich von RT bzw. 350$700{ }^{\circ} \mathrm{C}$ in Inertgas ( $\mathrm{He} \mathrm{bzw} . \mathrm{N}_{2}$ ) bzw. sauerstoffreicher Atmosphäre (He bzw. $\mathrm{N}_{2}$ mit 12,5 Vol.- $\% \mathrm{O}_{2}$, im Folgenden kurz $\mathrm{He} / \mathrm{O}_{2}$ bzw. $\mathrm{N}_{2} / \mathrm{O}_{2}$ ) durchgeführt und der Einfluss unterschiedlicher $\mathrm{O}_{2}$ - und CO-Konzentration sowie von $\mathrm{H}_{2} \mathrm{O}$ und kohlenstoffhaltiger Spezies bestimmt. Die Untersuchung der Adsorptions/Desorptionsprozesse erfolgte mittels eines AutoChem II 2920 Chemisorption Analyzers (Micromeritics Instrument Corp., USA) an kommerziellen Ptund ITO-Pulvern. Die Messungen umfassten sowohl Messungen der temperaturprogrammierten Desorption (TPD) als auch Puls-Chemisorptionsmessungen. Bei den TPD-Messungen wurde das Pulver nach Raumluftkontakt (1h, Raumtemperatur) mit einer Heizrate von $10 \mathrm{~K} / \mathrm{min}$ aufgeheizt und die Desorptionsprodukte mit Hilfe eines QuadrupolMassenspektrometer (ThermoStar GSD 301 T1, Pfeiffer Vacuum $\mathrm{GmbH}$, Deutschland) identifiziert. Bei den Puls-Chemisorptionsmessungen wurden in einen kontinuierlichen Trägergassstrom ( $\mathrm{He}$ bzw. $\left.\mathrm{He} / \mathrm{O}_{2}\right)$ bei konstanter Temperatur $\left(350-700^{\circ} \mathrm{C}, 50 \mathrm{~K}-\right.$ Abstufung) Prüfgaspulse (2000 ppm $\mathrm{CO}$ in $\mathrm{He}$ ) dosiert. Die Analyse erfolgte wiederum mit dem Massenspektrometer.

Zur Bestimmung der Gasart- und temperaturabhängigen elektrischen Leitfähigkeit wurden aus den 
Pulvern Dickschichtpasten hergestellt und im Siebdruckverfahren auf 3YSZ-Substraten abgeschieden. Nach einer Wärmebehandlung an Luft mit einer Peaktemperatur von $700^{\circ} \mathrm{C}$ weisen die porösen Schichten planare Abmessungen von $7 \times 2 \mathrm{~mm}^{2}$ und eine Dicke von ca. $10 \mu \mathrm{m}$ auf. Die elektrische Charakterisierung erfolgte mittels Gleichstrom-Widerstandsmessungen in einem Rohrofen mit einem vom Gasgemisch durchströmten Glasrohr-Reaktor. Für die Widerstandsmessung kam ein Typ 2400 Source Meter (Keithley Instruments/Tektronix, Inc., USA) zum Einsatz, die Temperatur wurde in unmittelbarer Nähe der Probe mittels eines Typ-S-Thermoelements bestimmt, welches durch einen Almemo 2390-3 Datenlogger (Ahlborn Mess- und Regelungstechnik, Germany) ausgelesen wurde. Wie bei den Adsorptions-/Desorptionsmessungen erfolgten sowohl Messungen nach Raumluftkontakt unter kontinuierlichem Aufheizen auf Peaktemperatur als auch Messungen in kontinuierlichem Gasstrom unter Zudosierung von $\mathrm{CO}$. Die Versuchsbedingungen wurden hinsichtlich Temperaturbereichen, Aufheizraten und weiterer Parameter vergleichbar mit jenen der Adsorptions-/Desorptionsmessungen gestaltet. Anhand einer für die Untersuchungen entwickelten potentiometrischen Messzelle auf Basis eines 10Sc1CeSZ-Festelektrolyts mit interner FeststoffReferenz erfolgte im selben Messplatz schließlich die Charakterisierung der Werkstoffe beim Einsatz als Messelektrode eines Festelektrolyt-Sensors.

\section{Ergebnisse}

Abb. 1 zeigt eine schematische Übersicht der Ergebnisse der Adsorptions-/Desorptionsmessungen an Pt-Pulver im Vergleich zu Literaturdaten [5-9]. Während am vorliegenden Pulver die Desorption von Sauerstoff, Wasser und $\mathrm{CO}_{2}$ zwischen Raum-

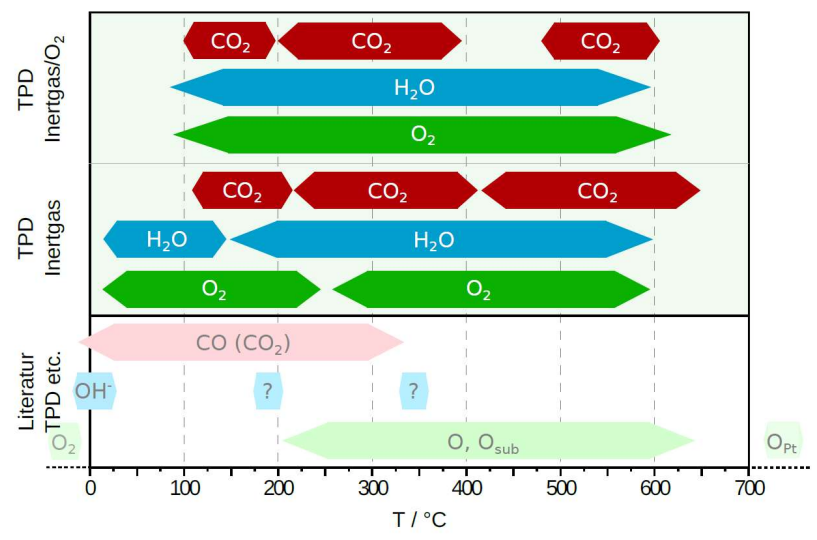

Abb. 1. Pt-Pulver, bei TPD-Untersuchung detektierte Gasspezies im Vergleich zu Literaturdaten. temperatur bzw. $100{ }^{\circ} \mathrm{C}$ und ca. $600{ }^{\circ} \mathrm{C}$ beobachtet wird, weichen die Literaturangaben sowohl qualitativ als auch quantitativ erheblich ab. Insbesondere die Desorption von $\mathrm{H}_{2} \mathrm{O}$ ist in der Literatur für Temperaturen oberhalb von $0{ }^{\circ} \mathrm{C}$ nur im Zusammenhang mit einer Ko-Adsorption mit $\mathrm{O}_{2}$ und $\mathrm{H}_{2}$ dokumentiert. In Abb. 2 ist eine schematische Übersicht der Ergebnisse der Adsorptions-/Desorptions- sowie der Widerstandsmessungen an ITO dargestellt. Die vergleichenden Literaturdaten wurden sowohl anhand von ITO, als auch anhand der konstituierenden Oxide $\mathrm{In}_{2} \mathrm{O}_{3}$ und $\mathrm{SnO}_{2}$ bestimmt [10-19].

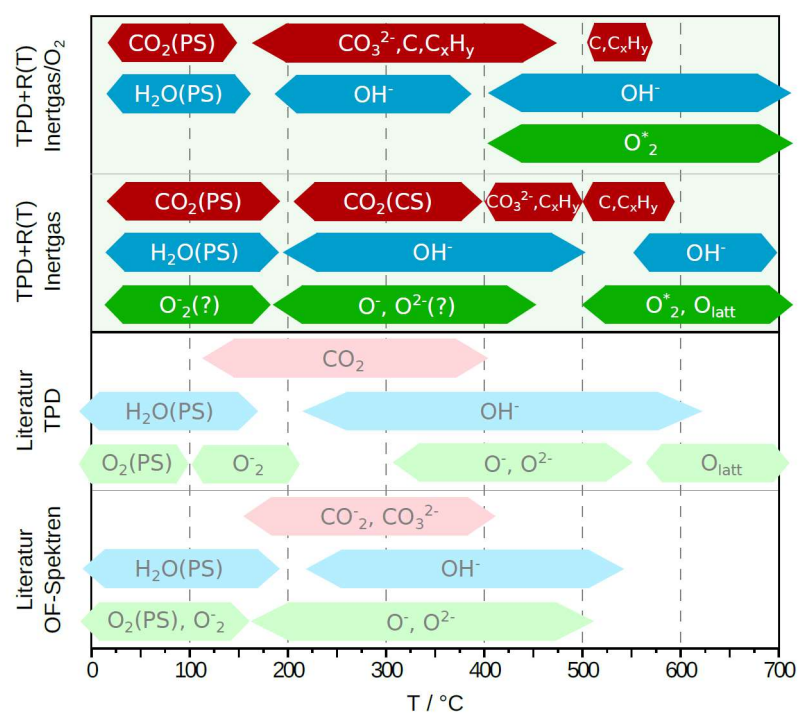

Abb. 2. ITO, anhand Adsorptions-/Desorptions- und Widerstandsmessungen bestimmte Gasspezies im Vergleich zu Literaturdaten.

Die Ergebnisse zeigen neben Sauerstoffspezies eine Vielzahl Wasser-verwandter und kohlenstoffhaltiger Spezies, deren Desorption auch bei Temperaturen von mehr als $600{ }^{\circ} \mathrm{C}$ noch beobachtet werden kann. Im Bereich oberhalb von $400{ }^{\circ} \mathrm{C}$ sind deutliche Abweichungen der Messergebnisse zu den Literaturdaten erkennbar, insbesondere für kohlenstoffhaltige Spezies jedoch auch im Bereich zwischen Raumtemperatur und $400^{\circ} \mathrm{C}$.

Abb. 3 zeigt die Zellspannung der potentiometrischen Messzelle mit Pt vs. ITO-Messelektrode als Funktion des Sauerstoffpartialdrucks. Während die Messdaten beider Elektroden bei $650{ }^{\circ} \mathrm{C}$ sehr gut übereinstimmen und die für Nernstsches Verhalten typische Signalabhängigkeit von $\ln \left(\mathrm{pO}_{2}\right)$ zeigen, steigt die Abweichung bei niedrigen Temperaturen für geringe Sauerstoffpartialdrücke stark an. 


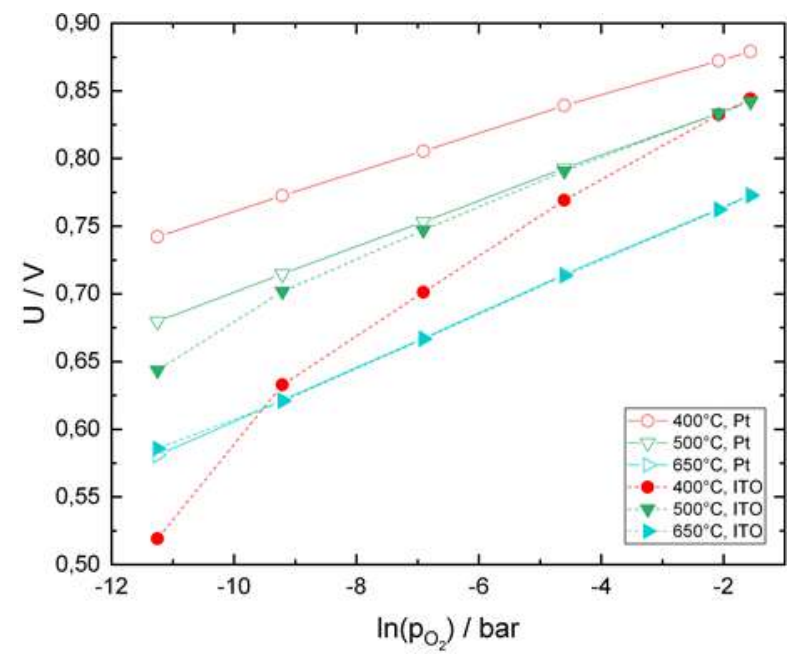

Abb. 3. Zellspannung U der potentiometrischen Messzelle mit Pt- vs. ITO-Messelektrode als Funktion des Sauerstoffpartialdrucks in $\mathrm{N}_{2}$ für ausgewählte Temperaturen

In Abb. 4 ist die CO-Empfindlichkeit beider Elektroden in $\mathrm{N}_{2}$ mit $12,5 \mathrm{Vol}$. $\% \mathrm{O}_{2}$ für verschiedene Temperaturen gegenübergestellt. In der Legende sind die entsprechenden Basisspannungen ohne COBeaufschlagung angegeben. Während sich die COEmpfindlichkeit der Zellen mit Pt- und ITO-Messelektrode bei $650{ }^{\circ} \mathrm{C}$ nur bei hohen $\mathrm{c}_{\mathrm{co}}$ geringfügig unterscheidet, ist sie für die ITO-Messelektrode bei $400{ }^{\circ} \mathrm{C}$ um den Faktor 2-3 gegenüber der PtMesselektrode erhöht.

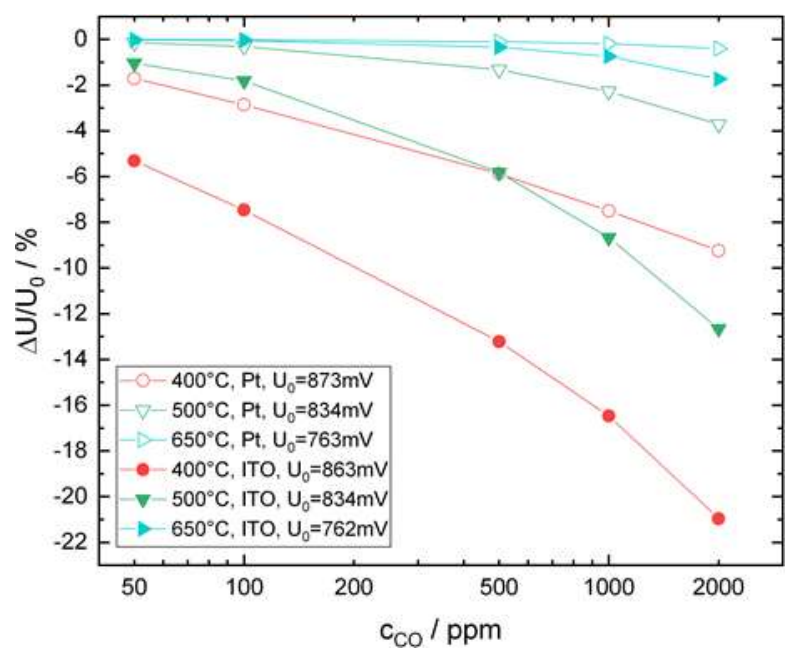

Abb. 4. Zellspannung $U$ der potentiometrischen Messzelle mit Pt- vs. ITO-Messelektrode als Funktion der CO-Konzentration in $\mathrm{N}_{2}+12,5$ Vol.- $\% \mathrm{O}_{2}$ für ausgewählte Temperaturen

\section{Diskussion}

Adsorptions/Desorptions- und elektrische Widerstandsmessungen

Für die Abweichungen der Messergebnisse am PtPulver sind neben der Art und Struktur des verwendeten Pulvers auch die Bedingungen bei der Beladung mit den entsprechenden Spezies sowie die konkreten Parameter der TPD-Untersuchung verantwortlich. Während in der Literatur vorwiegend Untersuchungen an monokristallinen Proben dokumentiert sind, welche mit Sauerstoff oder anderen Testgasen bei Temperaturen deutlich unterhalb $0{ }^{\circ} \mathrm{C}$ beladen wurden, erfolgte am vorliegenden polykristallinen Pulver die Beladung bei Raumtemperatur an Umgebungsluft. Es wird davon ausgegangen, dass die detektierten $\mathrm{H}_{2} \mathrm{O}$-Spezies auf der Pt-Oberfläche aufgrund einer Ko-Adsorption mit Sauerstoff stärker gebunden sind und die Desorption daher bei deutlich höheren Temperaturen beobachtet wird, als in der Literatur dokumentiert [9]. Eine Chemisorption von $\mathrm{CO}_{2}$ auf Pt-Oberflächen wurde in der Literatur bisher nicht beschrieben, physisorbiertes $\mathrm{CO}_{2}$ desorbiert von Pt-Oberflächen üblicherweise bei erheblich niedrigeren Temperaturen. Es ist daher davon auszugehen, dass das detektierte $\mathrm{CO}_{2}$ aus der Oxidation kohlenstoffhaltiger Verunreinigungen der Pt-Oberfläche stammt, welche sich bei Raumluftkontakt ablagern. Neben Carbonaten und Carboxylaten können dies verschiedene Kohlenwasserstoffe, jedoch auch graphitischer Kohlenstoff sein. Die verschiedenen Temperaturbereiche der $\mathrm{CO}_{2}$-Desorption deuten auf unterschiedliche kohlenstoffhaltige Spezies hin, eine konkrete Zuordnung bzw. Identifikation ist anhand der Messergebnisse jedoch nicht möglich.

Die Ergebnisse der Adsorptions-/Desorptionsmessungen an ITO zeigen, dass die ITO-Oberfläche in einem weiten Temperaturbereich durch $\mathrm{H}_{2} \mathrm{O}$ bzw. $\mathrm{OH}-G$ ruppen sowie kohlenstoffhaltige Adsorbate kontaminiert ist. Eine Zuordnung der Temperaturbereiche der Desorption zu konkreten Spezies ist anhand der Kombination mit den Ergebnissen der elektrischen Widerstandsmessungen möglich. Demnach liegen Wasser und $\mathrm{CO}_{2}$ im Bereich zwischen Raumtemperatur und $200{ }^{\circ} \mathrm{C}$ in physisorbierter Form auf der Oberfläche vor. Oberhalb von $200^{\circ} \mathrm{C}$ sind auf der ITO-Oberfläche vorwiegend $\mathrm{OH}$-Gruppen anzutreffen, welche bei höheren Temperaturen unter Freisetzung von Sauerstoff rekombinativ desorbieren [16]. Entsprechende Desorptionspeaks sind noch bei Temperaturen von bis $\mathrm{zu} 70{ }^{\circ} \mathrm{C}$ zu beobachten. Als kohlenstoffhaltige Adsorbate kommen im Temperaturbereich bis ca. $400^{\circ} \mathrm{C}$ vor allem Carbonate und Carboxylate, bei höheren Temperaturen jedoch auch Kohlenwasserstoffe und graphitischer 
Kohlenstoff in Betracht. Diese Spezies zeigen im Temperaturbereich von $350-450{ }^{\circ} \mathrm{C}$ eine teilweise gehemmte Desorption bzw. Zersetzung zu $\mathrm{CO}_{2}$, welche zu einer Beeinträchtigung der Adsorption von Sauerstoff und potentiellen Zielgasen, wie beispielsweise $\mathrm{CO}$, führt.

\section{Potentiometrische Messungen}

Die deutlichen Abweichungen der Zellspannungen der Messzellen mit Pt- und ITO-Messelektrode bei niedrigen Temperaturen bzw. geringen Sauerstoffpartialdrücken werden auf eine unvollständige Gleichgewichtseinstellung zwischen Sauerstoffmolekülen im Messgas, den Sauerstoffleerstellen im Festelektrolyt und Elektronen in der ITO-Messelektrode an der Dreiphasengrenze (triple phase boundary, TPB) zurückgeführt. Ein Vergleich mit den Ergebnissen der Adsorptions-/Desorptions- sowie der Widerstandsmessungen legt nahe, dass auch hier die Beeinträchtigung der $\mathrm{O}_{2}$-Adsorption auf der ITO-Oberfläche eine Rolle spielt. Sauerstoffadsorption und -diffusion haben direkten Einfluss auf die Sauerstoffbedeckung der Elektrodenoberfläche. Steht die Elektrodenoberfläche in der Nähe der TPB nicht im Gleichgewicht mit der Gasphase, kann auch die elektrochemische Gleichgewichtseinstellung an der TPB und damit die Potentialbildung beeinträchtigt werden. Bei $650{ }^{\circ} \mathrm{C}$ ist die Sauerstoffbedeckung der Pt- und ITO-Oberflächen aufgrund zunehmender Sauerstoffdesorption zwar generell geringer als bei niedrigen Temperaturen, die Gleichgewichtseinstellung zwischen Gasphase und ITO-Oberfläche wird jedoch nicht mehr so stark limitiert. Daher unterscheiden sich in diesem Temperaturbereich die Messergebnisse für beide Elektrodentypen nur wenig.

Die im Vergleich zur Pt-Messelektrode deutlich höhere CO-Empfindlichkeit der ITO-Messelektrode bei Temperaturen bis ca. $500{ }^{\circ} \mathrm{C}$ ist eine Folge zweier Effekte: Zum einen hat die beeinträchtigte Gleichgewichtseinstellung zwischen Gasphase und ITOOberfläche bzw. ITO-Oberfläche und dem Festelektrolyt wie im vorangegangenen Abschnitt beschrieben einen unmittelbaren Einfluss auf die Potentialbildung. Zum anderen führt die bei niedrigen Temperaturen geringere katalytische Aktivität der ITOOberfläche für die chemische Oxidation des $\mathrm{CO}$ da$\mathrm{zu}$, dass ein größerer Teil des $\mathrm{CO}$ die elektrochemisch aktive TPB-Region erreichen und zur Potentialbildung beitragen kann. Bei $650{ }^{\circ} \mathrm{C}$ ist der Unterschied zwischen beiden Elektrodentypen wiederum gering und die gemessene Zellspannung repräsentiert näherungsweise den GleichgewichtsSauerstoffpartialdruck.

\section{Literatur}

[1] Yadav, B. C.; Agrahari, Kaushlendra; Singh, Satyendra; Yadav, T. P.: Fabrication and Characterization of Nanostructured Indium Tin Oxide Film and Its Application as Humidity and Gas Sensors. In: Journal of Materials Science: Materials in Electronics 27 (2016), Mai, Nr. 5, S. 4172-4179. http://dx.doi.org/10.1007/s10854016-4279-x. DOI 10.1007/s10854-016-4279-x.

[2] Patel, N.G.; Makhija, K.K.; Panchal, C.J.: Fabrication of Carbon Dioxide Gas Sensor and Its Alarm System Using Indium Tin Oxide (ITO) Thin Films. In: Sensors and Actuators B: Chemical 21 (1994), September, Nr. 3 , S. 193-197. http://dx.doi.org/10.1016/09254005(94)01247-4. DOI 10.1016/0925-4005(94)01247-4

[3] Mbarek, Hedia; Saadoun, Moncef; Bessais, Brahim: Screen-Printed Tin-Doped Indium Oxide (ITO) Films for NH3 Gas Sensing. In: Materials Science and Engineering: C 26 (2006), März, Nr. 2-3, S. 500-504. http://dx.doi.org/10.1016/j.msec.2005.10.037. DOI 10.1016/j.msec.2005.10.037.

[4] Dietrich, Stefan: Funktionale Schichten für gassensorische Anwendungen, Technische Universität Dresden, Department of Materials Science and Engineering, Diss., 2018

[5] Gland, J.L.; Korchak, V.N.: The Adsorption of Oxygen on a Stepped Platinum Single Crystal Surface. In: Surface Science 75 (1980), Nr. 4, S. 733-750. http://dx.doi.org/10.1016/0039-6028(78)90190-5. DOI 10.1016/0039-6028(78)90190-5

[6] Derry, G.N.; Ross, P.N.: High Coverage States of Oxygen Adsorbed on $\mathrm{Pt}(100)$ and $\mathrm{Pt}(111)$ Surfaces. In: Surface Science 140 (1984), Nr. 1, S. 165-180. http://dx.doi.org/10.1016/0039-6028(84)90389-3. DOI 10.1016/0039-6028(84)90389-3

[7] Steininger, H.; Lehwald, S.; lbach, H.: Adsorption of Oxygen on Pt(111). In: Surface Science 123 (1982), Nr. 1, S. 1-17. http://dx.doi.org/10.1016/00396028(82)90124-8. DOI 10.1016/0039-6028(82)901248

[8] Jaeger, Nils I.; Jourdan, Angelika L.; Schulz-Ekloff, Günter: Effect of the Size of Platinum Particles on the Chemisorption of Oxygen. In: Journal of the Chemical Society, Faraday Transactions 87 (1991), Nr. 8, S. 1251. http://dx.doi.org/10.1039/ft9918701251. DOI $10.1039 / \mathrm{ft} 9918701251$

[9] Netzer, F.P.; Kneringer, G.: The Adsorption of Hydrogen and the Reaction of Hydrogen with Oxygen on $\mathrm{Pt}$ (100). In: Surface Science 51 (1975), August, Nr. 2, S. 526-538.http://dx.doi.org/10.1016/0039-6028(75) 90399-4. DOI 10.1016/0039-6028(75)90399-4

[10] Yamazoe, Noboru; Fuchigami, Jun; Kishikawa, Masato: Interactions of Tin Oxide Surface with $\mathrm{O}_{2}, \mathrm{H}_{2} \mathrm{O}$ and $\mathrm{H}_{2}$. In: Surface Science 86 (1979), S. 335-344. http://dx.doi.org/10.1016/0039-6028(79)90411-4. DOI 10.1016/0039-6028(79)90411-4

[11] Wiche, Gregor: Metalloxid-Gassensoren Mit Siliziumcarbid Micro-Hotplate, Technische Universität Berlin, Diss., 2007

[12] Gurlo, Alexander: Interplay between $\mathrm{O}_{2}$ and $\mathrm{SnO}_{2}$ : Oxygen lonosorption and Spectroscopic Evidence for Adsorbed Oxygen. In: ChemPhysChem 7 (2006), Oktober, Nr. 10, S. 2041-2052. 
http://dx.doi.org/10.1002/cphc.200600292.

DOI 10.1002/cphc. 200600292.

[13] Ayeshamariam, A.; Kashif, M.; Bououdina, M.; Hashim, U.; Jayachandran, M.; Ali, M.E.: Morphological, Structural, and Gas-Sensing Characterization of Tin-Doped Indium Oxide Nanoparticles. In: Ceramics International 40 (2014), Januar, Nr. 1, S. 1321-1328. http://dx.doi.org/10.1016/j.ceramint.2013.07.012. DOI 10.1016/j.ceramint.2013.07.012.

[14] Batzill, M; Diebold, U: The Surface and Materials Science of Tin Oxide. In: Progress in Surface Science 79 (2005), Nr. 2-4, S. 47-154 http://dx.doi.org/10.1016/j.progsurf.2005.09.002. DOI 10.1016/j.progsurf.2005.09.002.

[15] Afshar, Maziar; Preiss Elisabeth M.; Sauerwald, Tilman; Rodner, Marius; Feili, Dara; Straub, Martin; König, Karsten; Schütze, Andreas; Seidel, Helmut: Indium-Tin-Oxide Single-Nanowire Gas Sensor Fabricated via Laser Writing and Subsequent Etching. In: Sensors and Actuators B: Chemical 215 (2015), August, S. 525535. http://dx.doi.org/10.1016/j.snb.2015.03.067. DOI 10.1016/j.snb.2015.03.067.

[16] Egashira, Makoto; Nakashima, Masayo; Kawasumi, Shohachi; Selyama, Tetsuro: Temperature Programmed Desorption Study of Water Adsorbed on Metal Oxides. 2. Tin Oxide Surfaces. In: The Journal of Physical Chemistry 85 (1981), Dezember, Nr. 26, S. 4125\{4130. http://dx.doi.org/10.1021/j150626a034. DOI 10.1021/j150626a034.

[17] Sänze, Sandra: Synthese, Charakterisierung Und in Situ-Spektroskopische Studie Des Ethanol- Gassensormechanismus von Indiumoxid, Technische Universität Darmstadt, Diss., 2014

[18] Donley, Carrie; Dunphy, Darren; Paine, David; Carter, Chet; Nebesny, Ken; Lee, Paul; Alloway, Dana; Armstrong, Neal R.: Characterization of Indium-Tin Oxide Interfaces Using X-Ray Photoelectron Spectroscopy and Redox Processes of a Chemisorbed Probe Molecule: Effect of Surface Pretreatment Conditions. In: Langmuir 18 (2002), Januar, Nr. 2, S. 450-457. http://dx.doi.org/10.1021/la011101t. 10.1021/la011101t.

[19] Detweiler, Zachary M.; Wulfsberg, Steven M.; Frith, Matthew G.; Bocarsly, Andrew B.; Bernasek, Steven L.: The Oxidation and Surface Speciation of Indium and Indium Oxides Exposed to Atmospheric Oxidants. In: Surface Science 648 (2016), Juni, S. 188-195. http://dx.doi.org/10. 1016/j.susc.2015.10.026. DOI 10.1016/j.susc.2015.10.026. 